\title{
miR-222 isoforms are differentially regulated by type-I interferon
}

\author{
CHARLOTTE NEJAD, ${ }^{1,2}$ KATHERINE A. PILLMAN, ${ }^{3,4}$ KATHERINE J. SIDDLE, ${ }^{5}$ GENEVIÈVE PÉPIN, ${ }^{1,2}$ \\ MINNA-LIISA ÄNKÖ, ${ }^{6,7}$ CLAIRE E. MCCOY, ${ }^{2,8}$ TRAUDE H. BEILHARZ, ${ }^{7,9}$ LLUÍS QUINTANA-MURCI, ${ }^{10}$ \\ GREGORY J. GOODALL, ${ }^{3,11,12}$ CAMERON P. BRACKEN, ${ }^{3,11,12}$ and MICHAEL P. GANTIER ${ }^{1,2}$ \\ ${ }^{1}$ Centre for Innate Immunity and Infectious Diseases, Monash Institute of Medical Research, Monash University, Clayton, Victoria 3168 , Australia \\ ${ }^{2}$ Department of Molecular and Translational Science, Monash University, Clayton, Victoria 3168, Australia \\ ${ }^{3}$ Centre for Cancer Biology, SA Pathology and University of South Australia, Adelaide, South Australia 5000, Australia \\ ${ }^{4}$ ACRF Cancer Genomics Facility, Centre for Cancer Biology, SA Pathology, Adelaide, South Australia 5000, Australia \\ ${ }^{5}$ Department of Organismic and Evolutionary Biology, FAS Center for Systems Biology, Harvard University, Cambridge, Massachusetts 02138, USA \\ ${ }^{6}$ Department of Anatomy and Developmental Biology, Monash University, Victoria 3800, Australia \\ ${ }^{7}$ Development and Stem Cells Program, Biomedicine Discovery Institute, Monash University, Victoria 3800, Australia \\ ${ }^{8}$ Molecular and Cellular Therapeutics, Royal College of Surgeons in Ireland, Dublin 2, Ireland \\ ${ }^{9}$ Department of Biochemistry and Molecular Biology, Monash University, Melbourne, Victoria 3800, Australia \\ ${ }^{10}$ Institut Pasteur, Unit of Human Evolutionary Genetics, CNRS URA3012, Paris 75015, France \\ ${ }^{11}$ Department of Medicine, University of Adelaide, Adelaide, South Australia 5005, Australia \\ ${ }^{12}$ School of Molecular and Biomedical Science, University of Adelaide, Adelaide, South Australia 5005, Australia
}

\begin{abstract}
Endogenous microRNAs (miRNAs) often exist as multiple isoforms (known as "isomiRs") with predominant variation around their $3^{\prime}$-end. Increasing evidence suggests that different isomiRs of the same family can have diverse functional roles, as recently demonstrated with the example of miR-222-3p $3^{\prime}$-end variants. While isomiR levels from a same miRNA family can vary between tissues and cell types, change of templated isomiR stoichiometry to stimulation has not been reported to date. Relying on small RNA-sequencing analyses, we demonstrate here that miR-222-3p $3^{\prime}$-end variants $>23$ nt are specifically decreased upon interferon (IFN) $\beta$ stimulation of human fibroblasts, while shorter isoforms are spared. This length-dependent dynamic regulation of long miR-222-3p $3^{\prime}$-isoforms and $>40$ other miRNA families was confirmed in human monocyte-derived dendritic cells following infection with Salmonella Typhimurium, underlining the breadth of $\mathbf{3}^{\prime}$-length regulation by infection, beyond the example of miR-222-3p. We further show that stem-loop miRNA Taqman RT-qPCR exhibits selectivity between $3^{\prime}$-isoforms, according to their length, and that this can lead to misinterpretation of results when these isoforms are differentially regulated. Collectively, and to our knowledge, this work constitutes the first demonstration that the stoichiometry of highly abundant templated 3 '-isoforms of a same miRNA family can be dynamically regulated by a stimulus. Given that such 3 '-isomiRs can have different functions, our study underlines the need to consider isomiRs when investigating miRNA-based regulation.
\end{abstract}

Keywords: microRNA isoform; isomiR; interferon; stem-loop RT-qPCR; Taqman miRNA assays

\section{INTRODUCTION}

miRNAs are short 18-28 nt RNAs involved in the translational control of target messenger RNAs (mRNAs) (Ha and Kim 2014). During miRNA biogenesis, processing of precursor hairpins by Drosha and Dicer can lead to variation in the length of the $5^{\prime}$ - or $3^{\prime}$-end of a miRNA ( $\mathrm{Ha}$ and Kim 2014). Detection of such miRNA isoforms, referred to as templated isomiRs, was initially dismissed as an experimental artifact (Neilsen et al. 2012). Nonetheless, with the growing availability of small RNA-sequencing data sets, it is now clear that miRNA variants longer or shorter than the consen-

Corresponding author: michael.gantier@hudson.org.au

Article is online at http://www.rnajournal.org/cgi/doi/10.1261/rna. 064550.117 sus miRNA length in miRBase (Kozomara and GriffithsJones 2014) are often observed (Neilsen et al. 2012; Tan et al. 2014; Siddle et al. 2015; Karali et al. 2016; Juzenas et al. 2017; Telonis et al. 2017). Comparison of miRNA variants in 32 cancers recently found 7466 isomiRs from 807 miRNA arms (either $3 p$ or $5 p$, depending on their position in the precursor hairpin), with an average of approximately nine variants for each miRNA (Telonis et al. 2017). While 5' end variations of miRNAs have been linked to a functional impact on target mRNAs by changing their seed region

(c) 2018 Nejad et al. This article is distributed exclusively by the RNA Society for the first 12 months after the full-issue publication date (see http:// rnajournal.cshlp.org/site/misc/terms.xhtml). After 12 months, it is available under a Creative Commons License (Attribution-NonCommercial 4.0 International), as described at http://creativecommons.org/licenses/by-nc/4.0/. 
(Tan et al. 2014), the majority of isomiRs are $3^{\prime}$-end templated modifications (Neilsen et al. 2012; Siddle et al. 2015; Karali et al. 2016). Recent evidence suggests that in addition to contributing to the overall function of a miRNA family, $3^{\prime}$-end length variations can have different biological functions (Yu et al. 2017). As such, transfected miR-222-3p $>22$ nt $3^{\prime}$-end variants were found to control apoptosis through the PI3K-AKT pathway, while shorter miR-222-3p variants did not (Yu et al. 2017).

Several miRNAs are dynamically regulated upon stimulation of an innate immune response by pathogens (O'Neill et al. 2011). A recent study of human monocyte-derived dendritic cells (DCs) infected with several types of bacteria, including Salmonella enterica serovar Typhimurium, reported that pathogens have the capacity to influence the repertoire of templated miRNA isoforms (Siddle et al. 2015). This suggests that isomiR levels are not static and can be dynamically changed upon stimulation. Type-I IFNs are an essential effector of the immune response to pathogens, controlling expression of more than 2000 genes (Rusinova et al. 2013). The levels of several miRNAs have previously been shown to be regulated by type-I IFN stimulation (Sehgal et al. 2015), with the example of miR-221-3p and miR-222-3p that are decreased due to the activity of the IFN-inducible $3{ }^{\prime}-5^{\prime}$ exonuclease PNPT1 (Das et al. 2010). Whether type-I IFN stimulation modulates the repertoire of miRNA isoforms is however not known.

In the present work, we discovered that type-I IFN stimulation selectively decreases the levels of longer miR-221-3p and miR-222-3p isoforms, while sparing shorter ones. Such isomiR-specific dynamic regulation was not limited to these miRNAs and was also seen after infection by $S$. Typhimurium on more than 40 miRNA families, and could be detected by stem-loop miRNA Taqman RT-qPCR.

\section{RESULTS AND DISCUSSION}

Salmonella Typhimurium infection induces type-I IFN production, among other cytokines, in phagocytes (Robinson et al. 2012). Given that type-I IFN treatment modulates miRNA levels (Sehgal et al. 2015), we hypothesized that some of the effects observed on the cellular isomiR repertoire during S. Typhimurium infection (Siddle et al. 2015), could in fact pertain to type-I IFN activity. Analysis of miRNA isoform levels in human fibroblasts stimulated with IFN- $\beta$ by small RNA sequencing (RNA-seq) revealed a set of highly abundant miR-222-3p and miR-221-3p isoforms that were strongly decreased after stimulation (Fig. 1A; Supplemental Table S1). The decrease was limited to their longer $3^{\prime}$-isoforms as the shorter isoforms were rather increased (Fig. $1 \mathrm{~B}, \mathrm{C})$, and was independent on variation in proportions of nontemplated $3^{\prime}$-end bases. At least 12 additional miRNA families displayed a similar response to IFN- $\beta$, supporting the selective inducible decrease of a set of $3^{\prime}$-isomiRs by IFN- $\beta$ (Fig. 1D; Supplemental Table S1).
The effect of type-I IFN on miR-221-3p and miR-222-3p has previously been linked to their degradation by the type-I IFN-inducible $3^{\prime}-5^{\prime}$ exonuclease PNPT1, rather than transcriptional/processing regulation (Das et al. 2010). To rule out an effect of IFN- $\beta$ on a processing event, we measured the abundance of a synthetic 23 nt miR-221-3p transfected in human fibroblasts, with or without IFN- $\beta$ stimulation (Fig. 1E), by northern blot. The synthetic 23 nt miR-221$3 p$ miRNA was decreased upon IFN- $\beta$ treatment (Fig. $1 \mathrm{E})$, indicating that the action of IFN- $\beta$ stimulation operated downstream from miRNA maturation. In addition, we did not observe smaller size products after IFN- $\beta$ treatment, suggesting that the longer isomiRs were not trimmed into shorter ones, although warranting further studies to firmly establish this point. Critically, transfection of a synthetic 23 nt miR-221-3p with a mutated $3^{\prime}$-end failed to reproduce this effect upon IFN- $\beta$ stimulation (Fig. 1F), pointing to a role for the $3^{\prime}$-end of this miR-221-3p isoform in its inducible decrease, independent of precursor miRNA processing.

To determine whether the length-selective effect on isomiR described above was a phenomenon restricted to human fibroblasts treated with IFN- $\beta$, we reanalyzed the published small RNA-seq data set of monocyte-derived dendritic cells following $S$. Typhimurium infection (Siddle et al. 2015). Templated $3^{\prime}$-isoforms were assessed on the basis of their length from the same $5^{\prime}$-end, rather than their consensus (or canonical) miRBase annotation. The 23 and 24 nt variants of miR-221-3p and miR-222-3p, respectively, were abundant forms of these miRNAs and were decreased upon infection, while shorter isoforms were induced (Fig. $2 \mathrm{~A}$ ). It should be noted that beyond a role for IFN, the effect of $S$. Typhimurium on the increased levels of these shorter isoforms was likely impacted by NF- $\kappa \mathrm{B}$ activation, which has been shown to promote expression of the miR-221/222 primary transcript (Galardi et al. 2011). A similar trend was seen for all bacterial infections analyzed (Mycobacterium tuberculosis, Mycobacterium bovis BCG, and Yersinia pseudotuberculosis - not shown). Surprisingly, the effect of infection on longer isomiRs was more generalized than what we observed upon IFN- $\beta$ treatment of fibroblasts, and widely observed on isomiRs longer than $22 \mathrm{nt}$ (Fig. 2B). When restricting our analyses to miRNA families with at least five consecutive 3 -isoforms (in the range of 19-24 nt), we observed that 48 out of 58 miRNA families had selective decreased expression of the $>22$ nt isoforms, while isoforms $<21 \mathrm{nt}$ were increased in most cases (Fig. 2C). While miRNA trimming of these longer isoforms into shorter ones could have been at play in this context, it is also likely that the effect on isomiR stoichiometry here resulted from a compounded effect of transcriptional and processing regulation, in addition to potential selective isomiR degradation. Critically, the vast array of miRNAs families impacted stresses further the need to take isomiRs into account when investigating miRNA levels. 

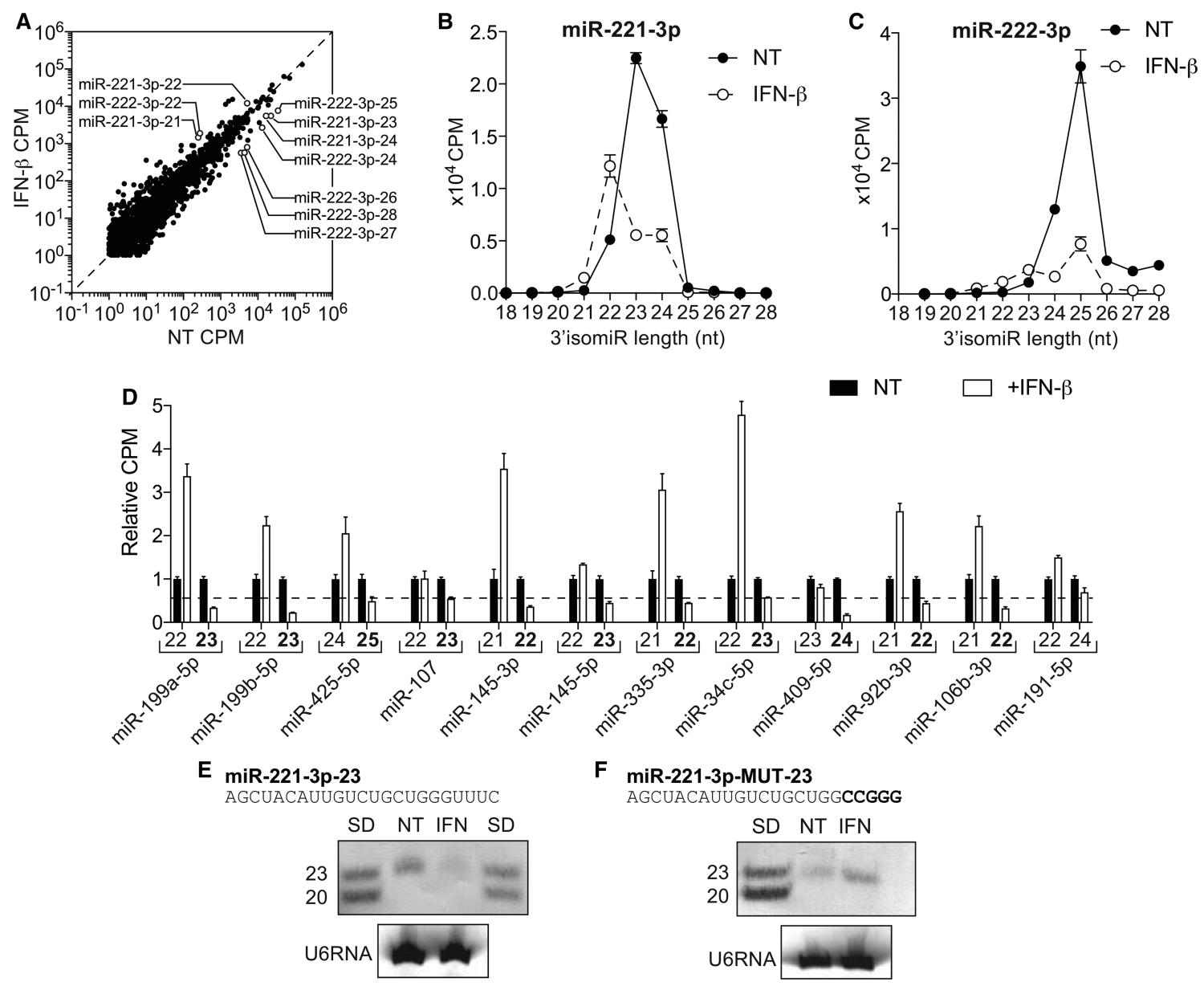

FIGURE 1. IFN- $\beta$-dependent decrease of long 3 '-isomiRs in human fibroblasts. (A) Human fibroblasts were treated with $1000 \mathrm{IU} / \mathrm{mL}$ of recombinant human IFN- $\beta$ for $24 \mathrm{~h}$ prior to total RNA purification. Small RNA-seq analysis was performed in biological triplicate, and read count per million (CPM) calculated for each isoform, based on their length from the canonical $5^{\prime}$-end. The data shown are averaged from biological triplicate, and limited to $17953^{\prime}$-isomiRs $<30 \mathrm{nt}$ and $>1$ CPM for each condition (see Supplemental Table S1; Materials and Methods). (NT) Nontreated. (B,C) Detailed analysis of the $3^{\prime}$-end isoforms of miR-221-3p $(B)$ and miR-222-3p $(C)$. (D) CPMs of 12 miRNA families, for the predominant $3^{\prime}$-isomiR in bold, and $1 \mathrm{nt}$ shorter variant, are shown relative to the NT condition (for miR-191-5p, the 2 nt shorter variant is shown). (B-D) Analyses are based on the samples from $A$ and mean \pm SEM is shown. $(E, F)$ Human fibroblasts were transfected with $1.6 \mathrm{nM}$ of the indicated synthetic miR-221-3p duplex for $24 \mathrm{~h}$, prior to treatment with or without $1000 \mathrm{IU} / \mathrm{mL}$ of recombinant human IFN- $\beta$ for an additional $24 \mathrm{~h}$. Total RNA was extracted and synthetic $23 \mathrm{nt}$ miR-221-3p and U6 RNA detected by northern blot. miR-221-3p-MUT-23 contains a mutation of the last five bases at the $3^{\prime}$-end. Twenty-three and $20 \mathrm{nt}$ synthetic miR-221-3p were loaded as size controls $(\mathrm{SD}) .(E, F)$ Data shown are representative of a minimum of two independent experiments. (NT) Nontreated.

Having established the dynamic effect of IFN- $\beta$ and bacterial infection on miR-222-3p, we next investigated the feasibility of using RT-qPCR techniques to rapidly measure the breadth of our observation across cell types and stimuli, without relying on expensive small RNA-seq analyses. Previous studies have suggested that stem-loop Taqman RT-qPCR, which remains a gold standard in miRNA detection, may not be selective for $3^{\prime}$-isomiRs with \pm 1 base variation ( $\mathrm{Wu}$ et al. 2007; Schamberger and Orban 2014; Magee et al. 2017). We noted that two predesigned Taqman assays were available for miR-222-3p, designed to target the $21 \mathrm{nt}$ and $24 \mathrm{nt}$ isomiRs, due to the re-annotation of miR-222-3p as a $21 \mathrm{nt}$ miRNA in miRBase V10 (Fig. 3A). Comparison of the $21 \mathrm{nt}$ and the $24 \mathrm{nt}$ miR-222-3p assays on synthetic
miR-222-3p isoforms revealed that the $21 \mathrm{nt}$ isoform was selectively detected by the $21 \mathrm{nt}$ assay, and only marginally amplified by the $24 \mathrm{nt}$ assay (Fig. 3B-at least approximately $10^{6}$ fold less well than the $21 \mathrm{nt}$ isomiR primers). Similarly, the 24 $\mathrm{nt}$ isoform was preferentially detected by the $24 \mathrm{nt}$ assay, albeit with a lower level of specificity since it could also be amplified by the $21 \mathrm{nt}$ assay (Fig. 3B). Comparable findings were replicated with miR-221-3p 20 and 23 nt isoforms (Fig. 3A, B). Further analysis of synthetic miR-222-3p isoforms ranging from 21 to $25 \mathrm{nt}$ showed that the best amplification target of the $21 \mathrm{nt}$ Taqman assay was in fact the $22 \mathrm{nt}$ isoform, and that the assay had increased selectivity for the $22-23 \mathrm{nt}$ isoforms (Fig. 3C). The $24 \mathrm{nt}$ Taqman assay, on the other hand, best amplified the $25 \mathrm{nt}$ isoform, with selective 

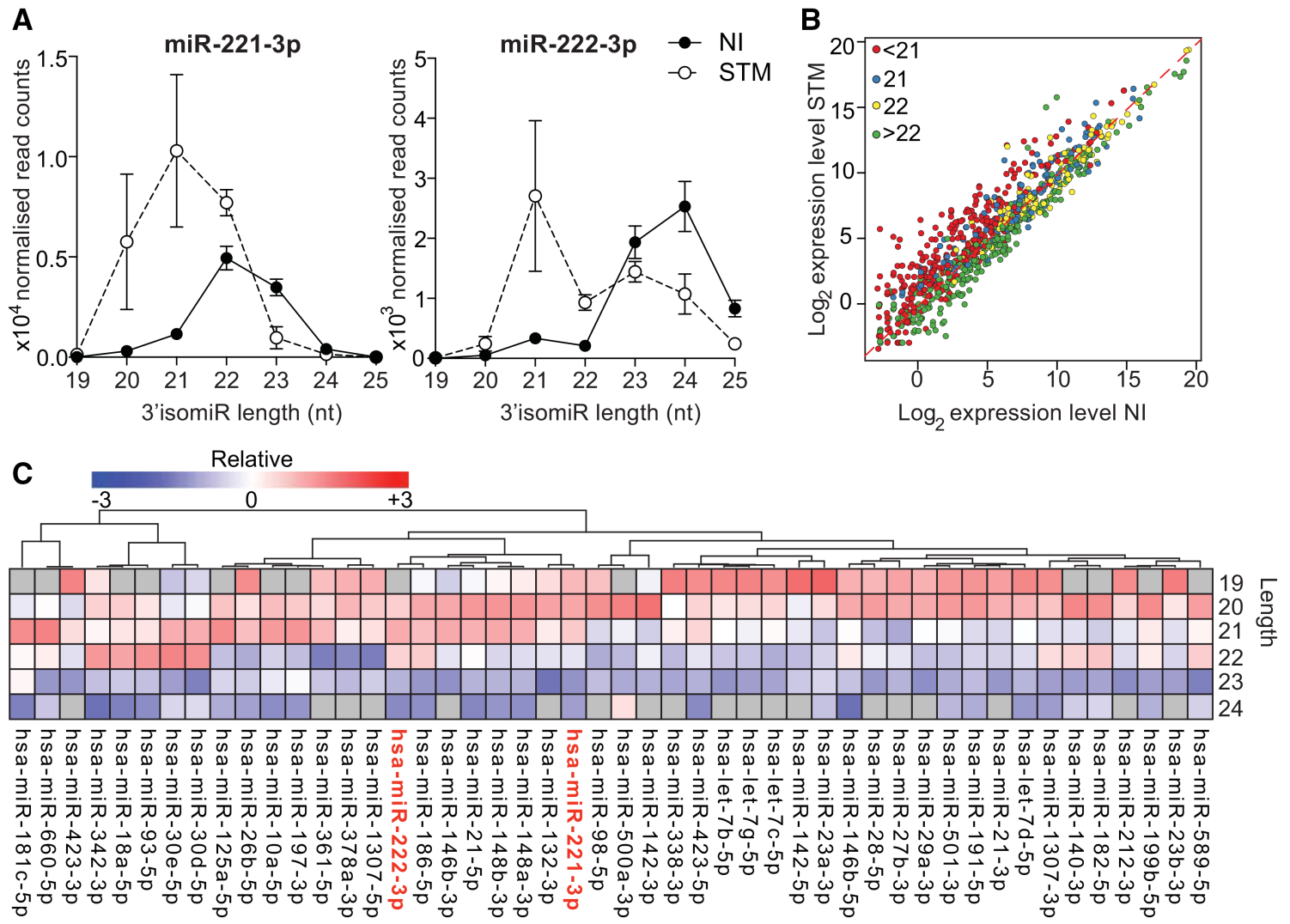

FIGURE 2. $3^{\prime}$-End length-dependent regulation of miRNA isoforms in $S$. Typhimurium infected DCs. (A) Detailed analysis of normalized read counts for the $3^{\prime}$-end isoforms of miR-221-3p and miR-222-3p in human monocyte-derived dendritic cells after $48 \mathrm{~h}$ infection with Salmonella Typhimurium (STM) analyzed by small RNA-seq. (NI) Noninfected. Data are averaged from six individuals (mean \pm SEM is shown), and 3'isomiRs restricted to templated isoforms. (B) $\log _{2}$ expression of 132 miRNA families in noninfected (NI) versus Salmonella Typhimurium (STM) infected DCs at $48 \mathrm{~h}$ (based on Supplemental Table S2). Each point represents the mean across six individuals from a condition, and is color-coded according to the miRNA length. $(C)$ Relative heatmap representation of $\log _{2}$ fold change of selected miRNA families at $48 \mathrm{~h}$ after Salmonella Typhimurium infection (see Materials and Methods). Gray indicates no significant detection by RNA sequencing. Selected miRNAs of interest are highlighted in red.

amplification of 23-25 nt isoforms over the 21-22 nt ones (Fig. 3C).

To test the capacity of the Taqman assays to distinguish between $3^{\prime}$-isomiRs in biological samples, we next analyzed the assays targeting miR-221-3p and miR-222-3p 20/23 and 21/ $24 \mathrm{nt}$ isoforms, respectively, in human fibroblasts stimulated with IFN- $\beta$ (Fig. 4A). In line with small RNA-seq analyses and an isoform selectivity of the Taqman assays, the RTqPCR approach showed an inverse response of long and short isomiRs of miR-221-3p and miR-222-3p to IFN- $\beta$, with the 23/24 Taqman assays being significantly decreased, while the 20/21 Taqman assays where rather induced (Fig. 4A). A similar response was reproduced in mouse bone marrow derived macrophages (BMDMs) (Fig. 4B), and time-course analysis of miR-222-3p isomiRs in human fibroblasts after IFN- $\beta$ treatment showed a progressive decrease with the 24 nt but not the $21 \mathrm{nt}$ Taqman assay with time $(17 \%$ at $4 \mathrm{~h}$, $36 \%$ at $8 \mathrm{~h}, 56 \%$ at $12 \mathrm{~h}$ and $78 \%$ at $24 \mathrm{~h}$ ), supporting the involvement of a selective enzymatic degradation of the longer isoforms (Fig. 4C). To broaden our observations, we also an- alyzed the levels of four microRNAs for which the Taqman assays had been predesigned to target longer isoforms $(\geq 22$ nt-see Materials and Methods), decreased in human fibroblasts by IFN- $\beta$ according to our small RNA-seq analyses (Fig. 1; Supplemental Table S1). These RT-qPCR analyses confirmed the significant decrease with the miR-145-5p, miR-191-5p, miR-106b-3p, and miR-107 assays (Fig. 4D). However, the decrease of miR-92b-3p 22 nt was not validated by the Taqman assay, which was targeted to its $21 \mathrm{nt}$ isoform (probably compensating for the decrease of the $22 \mathrm{nt}$ ).

Critically, PNPT1 down-regulation by RNA interference attenuated the isomiR-dependent effect of IFN- $\beta$ on miR222-3p in human melanoma cells (MeWo cells) - in accord with precedent studies (Fig. 4E; Das et al. 2010). Western blot analysis of PNPT1 confirmed its induction at the protein level by IFN- $\beta$, and the inhibitory effect of the two small interfering RNAs (siRNAs) used in MeWo cells (Fig. 4F). The effect of IFN- $\beta$ and PNPT1 was also studied for miR-221$3 \mathrm{p} 23 \mathrm{nt}$ and the four additional miRNA assays, which were significantly decreased in human fibroblasts (Fig. 4G). The 


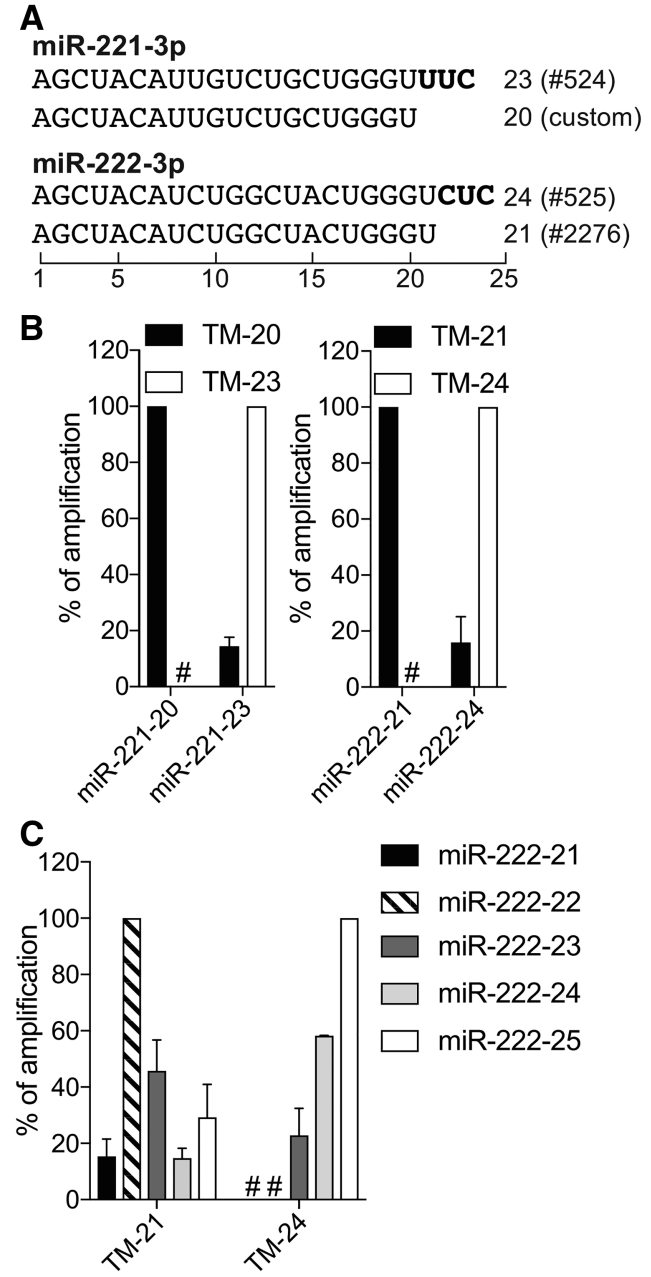

FIGURE 3. IsomiR-selectivity of Taqman RT-qPCR assays. (A) miR$221-3 p$ and miR-222-3p $3^{\prime}$-end isomiR sequences are provided, along with their respective Taqman assay reference. (B) Stem-loop Taqman RT-qPCR detection of synthetic miR-221-3p and miR-222-3p 20/23 and 21/24 variants, respectively. The Taqman assays are referred to as "TM" in the figure, while synthetic isomiRs are referred to as "miR," with their length appended. The amplification values are normalized per isomiR, to the lowest Cq value obtained and are displayed as percentages. (C) Stem-loop Taqman RT-qPCR detection of miR-222-3p $3^{\prime}$-isomiRs ranging from 21 to $25 \mathrm{nt}$. The amplification values are normalized per Taqman assay, to the lowest $\mathrm{Cq}$ value obtained for each assay and are displayed as percentages. (\#) Amplification values smaller than $0.1 \%$. $(A-C)$ Data shown are averaged from two independent RT-qPCRs (mean \pm SEM is shown).

long isoforms of miR-221-3p and miR-107 were significantly reduced by IFN- $\beta$ and involved PNPT1 activity since PNPT1 down-regulation inhibited their IFN- $\beta$-dependent decrease (Fig. 4G). Conversely, for the miR-145-5p and miR-191-5p assays, the IFN- $\beta$-dependent decrease was not impacted by the PNPT1 siRNA in MeWo cells, suggesting a PNPT1-independent activity of IFN- $\beta$ on these miRNA isoforms (Fig. $4 \mathrm{G})$. Furthermore, PNPT1 down-regulation in human fibroblasts failed to rescue the IFN- $\beta$-dependent decrease of the miR-222-3p 24 nt assay (not shown), underlining that the ef- fect of IFN- $\beta$ on long miR-222-3p isoforms is probably not restricted to PNPT1. Further studies are therefore warranted to help define whether additional actors contribute to this isoform-specific effect of IFN- $\beta$. Nonetheless, these experiments suggest that the effect of PNPT1 on the miR-222-3p, miR-221-3p, and miR-107 families may be restricted to their longer isomiRs. Collectively, our finding constitutes, to our knowledge, the first direct evidence that the stoichiometry of highly abundant templated 3 '-isoforms of a same miRNA family can be dynamically regulated by a stimulus.

Although thorough comparison between long and short miR-222-3p 3'-isomiR showed little difference on the regulation of the target levels (Yu et al. 2017), we note that the effect of type-I IFN on selected isomiRs impacted the overall intracellular concentration of miR-221-3p, miR-222-3p, miR199a/b-5p, miR-335-3p, miR-409-5p, miR-145-3p, and miR-145-5p by more than $40 \%$ (Table 1 ), thereby presumably decreasing the regulatory activities on their targets. Critically, the total intracellular levels of miR-222-3p, which is the second most abundant miRNA in human fibroblasts (Supplemental Table S1), were decreased by $\sim 70 \%$ upon type-I IFN-treatment (Table 1). Given that miR-222-3p can enhance NF- $\mathrm{kB}$ and STAT3 signaling (Galardi et al. 2011; Liu et al. 2014), the effect of IFN-treatment on the total intracellular levels of miR-222-3p may be part of the broad regulation of type-I IFN to limit the duration of inflammatory responses. In addition, recent evidence suggests that the $24 / 25$ nt 3 '-end miR-222-3p isoforms (but not the $21 \mathrm{nt}$ isoform) repress AKT expression, through transcriptional control of PIK3R3 expression. This effect of the $>23 \mathrm{nt}$ isoforms of miR-222-3p was proposed to relate to their enhanced nuclear localization compared to 20-22 nt isoforms, a trend globally observed for most miRNAs in their analyses (Yu et al. 2017). Since AKT activity facilitates antiviral responses (Kaur et al. 2008), the decrease of long miR-222-3p isoforms may potentiate the antiviral effects of type-I IFN, although clearly warranting further studies.

Our findings emphasize that the canonical length of a miRNA, currently defined in miRBase according to a landmark miRNA cloning study (Landgraf et al. 2007), is to be taken with caution as it introduces a direct bias in the assessment of $3^{\prime}$-addition or trimming, potentially masking relatively widespread $3^{\prime}$-length variations. In addition, a major consequence of such canonical length is that it is generally used to design miRNA-specific RT-qPCR primers and probes. To illustrate the impact that this design can have on result interpretation, we revisited a Taqman low density RT-qPCR array that we had previously carried out on BMDMs stimulated with bacterial lipopolysaccharide (LPS) (which activates Toll-like receptor 4 [TLR4] and induces type-I IFN production [Yamamoto et al. 2003]). According to the qPCR array analysis, miR-222-3p (detected with the $21 \mathrm{nt}$ isomiR assay), was induced more than twofold in the presence of the TLR4 agonist, while miR-221-3p (detected by the $23 \mathrm{nt}$ isomiR assay) was decreased more than twofold 
A
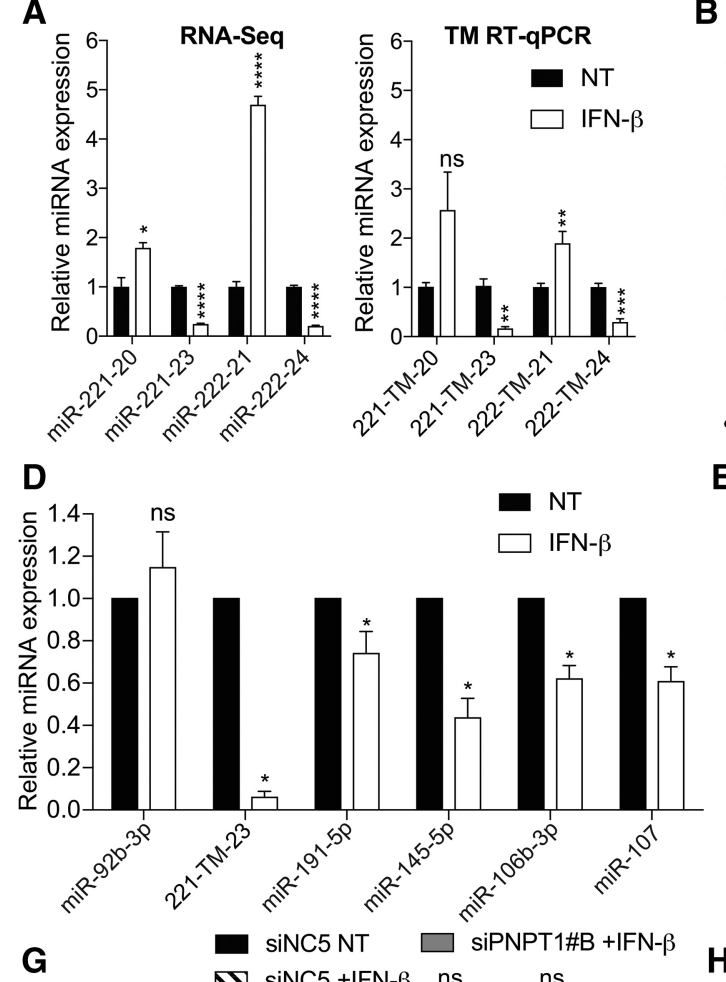

H
B

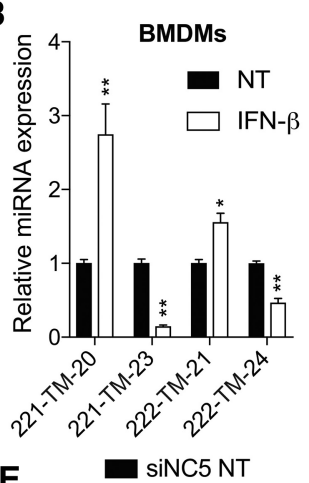

$\mathrm{E}$
C

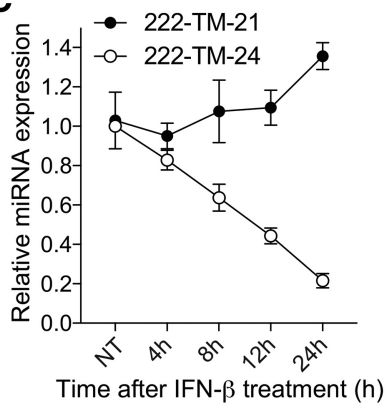

들 ${ }^{2.0} \square$ siNC5 +IFN- $\beta \square$ siPNPT1\#A +IFN- $\beta$

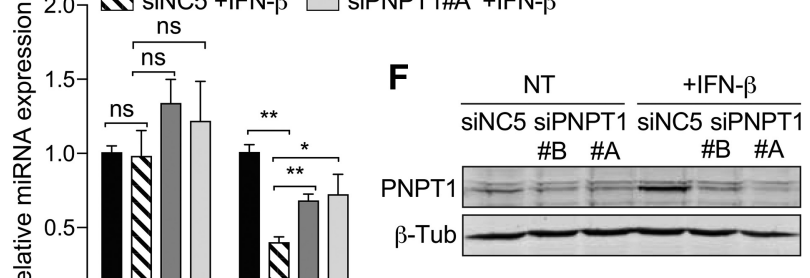
$\mathbf{N}$<smiles>C=[14CH][14CH]=[14CH]</smiles>
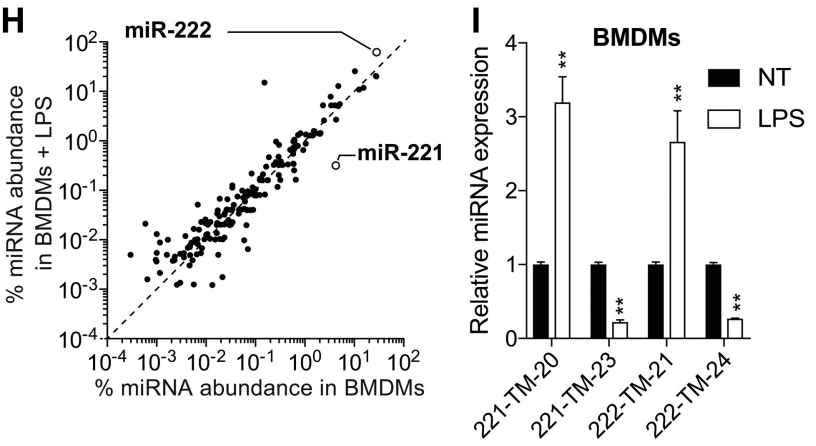

FIGURE 4. The isomiR-specific effect of IFN- $\beta$ can be detected by RT-qPCR. (A) Human fibroblasts were treated with $1000 \mathrm{IU} / \mathrm{mL}$ of recombinant human IFN- $\beta$ for $24 \mathrm{~h}$ prior to total RNA purification (similar to Fig. 1A). $3^{\prime}$-isomiR levels measured by stem-loop Taqman RT-qPCR were reported to U6 RNA, and are referred to as "TM" in the figure. Data from small RNA-seq (left) and RT-qPCR (right) approaches are shown relative to NT for each isoform selected. Data are averaged from three independent experiments (mean \pm SEM and unpaired $t$-tests are shown relative to NT condition for each isomiR/detection method). (B) Mouse BMDMs were treated with $1000 \mathrm{IU} / \mathrm{mL}$ of recombinant mouse IFN- $\beta$ for $24 \mathrm{~h}$ prior to total RNA purification. $3^{\prime}$-isomiR levels measured by stem-loop Taqman RT-qPCR were reported to sno202 RNA. Data are shown relative to NT for each isoform selected, and are averaged from three independent experiments in biological duplicate (mean \pm SEM and unpaired Mann-Whitney $U$-tests are shown). (C,D) Human fibroblasts were treated with $1000 \mathrm{IU} / \mathrm{mL}$ of recombinant human IFN- $\beta$ for indicated amount of time and the levels of miR222-3p isoforms $(C)$ or indicated miRNAs $(D)$ measured by stem-loop Taqman RT-qPCR as in $A$. $(C, D)$ Data are averaged from two independent experiments in biological duplicate (mean \pm SEM is shown). $(E-G)$ Human MeWo cells were transfected overnight with $10 \mathrm{nM}$ of nontargeting siRNA (siNC5) or siPNPT1\#A or \#B, before $24 \mathrm{~h}$ stimulation or not with $1000 \mathrm{IU}$ of recombinant human IFN- $\beta$. (E) Mature miR-222-3p isoform levels were quantified by RT-qPCR (data are shown relative to siNC5 NT condition). Data are averaged from two (siPNPT1\#A) or three (for siPNPT1\#B) independent experiments in biological duplicate (mean \pm SEM and unpaired Mann-Whitney $U$-tests are shown). (NT) Nontreated. $(F)$ PNPT1 protein levels analyzed by western blot (blots are representative of two independent experiments). ( $G$ ) Indicated miRNAs were quantified by stem-loop Taqman RT-qPCR (data are shown relative to siNC5 NT condition). Data are averaged from three independent experiments in biological duplicate (mean \pm SEM and unpaired Mann-Whitney $U$-tests are shown). (NT) Nontreated. (H) Taqman profiling of 335 mouse miRNAs was performed in BMDMs stimulated or not for $24 \mathrm{~h}$ with the TLR4 agonist LPS ( $100 \mathrm{ng} / \mathrm{mL}$ ) (one sample per condition). Cqs were used to determine the relative abundance of each miRNA to U6 (see Materials and Methods) and are shown as percentages. miR-222-3p is targeted by the Taqman assay \#2276, with selectivity to the short $21 \mathrm{nt}$ isomiR, while miR-221-3p is targeted by the Taqman assay \#524 with selectivity to the long $23 \mathrm{nt}$ isomiR. (I) BMDMs were stimulated with TLR4 ligand (100 ng/mL LPS) for $24 \mathrm{~h}$. 3'-isomiR levels measured by stem-loop Taqman RT-qPCR were reported to sno202 RNA. Data are shown relative to NT for each isoform selected, and are averaged from three independent experiments in biological duplicate (mean \pm SEM and unpaired Mann-Whitney $U$-tests are shown). $\left({ }^{*}\right) P \leq 0.05,\left({ }^{* *}\right) P \leq 0.01,\left({ }^{* * *}\right) P \leq 0.001,\left({ }^{* * * *}\right) P \leq$ 0.0001, (ns) not significant. 
TABLE 1. The impact of type-I IFN on total CPM of selected miRNA families

\begin{tabular}{llll}
\hline Name & NT & +IFN & \% decrease \\
\hline hsa-miR-21-5p & 253500.9921 & 231386.2117 & $\mathbf{8 . 7 2 3 7 4 5 1 2}$ \\
hsa-miR-222-3p & 63606.11933 & 18919.50666 & $\mathbf{7 0 . 2 5 5 2 0 9 9 4}$ \\
hsa-miR-221-3p & 45399.22706 & 25085.55987 & $\mathbf{4 4 . 7 4 4 5 2 2 1 1}$ \\
hsa-miR-199a-5p & 12180.23599 & 5795.980758 & $\mathbf{5 2 . 4 1 4 8 7 3 0 4}$ \\
hsa-miR-191-5p & 8792.480438 & 8227.508455 & 6.425626849 \\
hsa-miR-199b-5p & 6939.176193 & 2861.54255 & $\mathbf{5 8 . 7 6 2 5 0 3 3 6}$ \\
hsa-miR-145-5p & 4519.974523 & 2049.423398 & $\mathbf{5 4 . 6 5 8 5 1 8 8 1}$ \\
hsa-miR-425-5p & 1337.803154 & 1049.244955 & 21.5695559 \\
hsa-miR-92b-3p & 1277.162004 & 1168.352428 & 8.519637707 \\
hsa-miR-335-3p & 1088.469216 & 627.7423432 & $\mathbf{4 2 . 3 2 7 9 6 5 3 5}$ \\
hsa-miR-34c-5p & 788.148624 & $\mathbf{1 8 5 . 4 9 9 3 5 1 1 5}$ \\
hsa-miR-409-5p & 727.8664628 & 338.3462425 & $\mathbf{5 3 . 5 0 7 6 4 6 1}$ \\
hsa-miR-106b-3p & 573.0973398 & 5.504693113 \\
hsa-miR-107 & 400.2802677 & 518.6261964 & 18.28755571 \\
hsa-miR-145-3p & 278.7141124 & 327.0787908 & $\mathbf{4 1 . 5 6 4 7 7 2 1 1}$ \\
\hline
\end{tabular}

These results were obtained by adding the averaged CPMs (across three biological replicates) of all $3^{\prime}$-end isomiR variants detected for each miRNA family stated here. This estimates the total concentration of a miRNA family, independent of its isomiRs. Percent decrease of CPM after $24 \mathrm{~h}$ IFN treatment is provided, with miRNA families decreased by more than $40 \%$ highlighted in bold. The miR-21-5p family (white shading) was the most highly abundant family and is provided as a reference.

(Fig. 4E), suggesting a differential response of both miRNA to TLR4 stimulation. However, this interpretation was entirely biased by the isomiR-selectivity of the Taqman assays present on this low density RT-qPCR array, as confirmed by the individual analyses of miR-221-3p and miR-222-3p with both 20/23 and 21/24 nt isomiR-selective Taqman assays, respectively, in LPS-treated BMDMs (Fig. 4F).

Collectively, our findings establish the capacity of templated miRNA isoform intracellular levels to be differentially regulated upon stimulation. With the emerging capacity of isomiRs to exhibit differential functions and cellular localization, according to their $3^{\prime}$-end length (Yu et al. 2017), our study underlines the necessity to take isomiRs into account to obtain the full picture of miRNA regulatory activities. Informed knowledge about the design of the assays used to detect miRNAs is also essential, given that certain assays such as the stem-loop Taqman RT-qPCR assays can display isoform selectivity. However, such isoform selectivity could also be built upon to design a novel generation of miRNAbiomarker assays, for instance allowing the detection of cancer-specific isomiRs (Telonis et al. 2017).

\section{MATERIALS AND METHODS}

\section{Ethics statement}

The use of animals and experimental procedures were approved by the Monash Medical Centre Ethics Committee under reference MMCA2011/25.

\section{Cell culture}

For bone marrow derived macrophages (BMDMs), bone marrow was isolated from the femurs of wild type C57BL/6 mice, and differ- entiated in 20\% L929-cell-conditioned medium for $6 \mathrm{~d}$ at $37^{\circ} \mathrm{C}$ in a $5 \% \mathrm{CO}_{2}$ atmosphere, as previously described (Ferrand and Gantier 2016). Human hTERT BJ fibroblasts (referred to as human fibroblasts herein-gift from V. Hornung, Ludwig-MaximiliansUniversity) were grown in DMEM (Life Technologies) supplemented with $10 \%$ sterile fetal bovine serum (Life Technologies), $1 \mathrm{mM}$ sodium pyruvate and $1 \times$ antibiotic/antimycotic (Life Technologies) (referred to as complete DMEM). MeWo cells (gift from X. Zhang, Peter MacCallum Cancer Centre) were grown in RPMI 1640 medium (Life Technologies) supplemented with $20 \mathrm{mM}$ HEPES, 10\% sterile fetal bovine serum and $1 \times$ antibiotic/antimycotic (referred to as complete RPMI). Lipopolysaccharide (LPS) from E. coli Serotype O111: B4 (TLR4 agonist, Enzo Life Sciences), was used at indicated concentration. Recombinant mouse (Stifter et al. 2014) (gift from N.A. de Weerd and P.J. Hertzog, Hudson Institute) and human IFN- $\beta$ (Rebif, Merck Serono) were used at a final activity of $1000 \mathrm{IU} / \mathrm{mL}$.

\section{Reverse transcription quantitative real-time PCR (RT-qPCR)}

Total RNA was purified using the innuPREP MicroRNA Kit (Analytik Jena) or the GenElute Total RNA Purification Kit (Sigma-Aldrich). Stem-loop miRNA TaqMan assays (Applied Biosystems) were used according to the manufacturer's instructions, where 10-30 ng of total RNA or 3.2 fmol of synthetic miRNA was reverse transcribed with individual or pools of miRNA-specific reverse transcription primers. The manufacturer's references of the assays used are: miR-222-3p (\#525 - "222-TM-24" and \#2276 - "222TM-21"); miR-221-3p (\#524 - “221-TM-23”); miR-145-5p (\#2278) (23 nt isoform); miR-106b-3p (\#2380) (22 nt isoform); miR-191-5p (\#490) (22 nt isoform); miR-107 (\#443) (23 nt isoform); hsa-miR92b-3p (\#7028) (21 nt isoform). A custom miRNA Taqman assay targeted to the $20 \mathrm{nt}$ variant of miR-221-3p (based on AGCUACAUUGUCUGCUGGGU) (“221-TM-20”) was made by Applied Biosystems. The SensiFAST Probe Hi-ROX Kit (Bioline) was used, and fold changes in expression were calculated by the 
$2^{-\Delta \Delta \mathrm{Cq}}$ (Cq-quantification cycle) method using snoRNA202 (\#1232) and U6 RNA (\#1973) as reference small RNAs in mouse and human cells, respectively. For Figure 3, relative amplification of synthetic miRNAs was calculated using $2^{-\Delta \mathrm{Cq}}$ per line. Each RT-qPCR was carried out in technical duplicate.

\section{miRNA profiling}

TaqMan Array Rodent MicroRNAs (A Cards v2.0, Applied Biosystems) were used for the global detection of miRNAs (Fig. 4), as previously described (Gantier et al. 2011). The Cqs obtained for each miRNA were used to infer the abundance of each miRNA relative to that of the U6 short RNA. The relative level of each miRNA to U6 was then inferred using $2^{(-\Delta \mathrm{Cq})}$, and is shown as a percentage in Figure 4E.

\section{Synthetic RNAs}

Synthetic miRNAs were synthesized as single-stranded RNAs by Integrated DNA Technologies (IDT), and resuspended in duplex buffer (100 mM potassium acetate, $30 \mathrm{mM}$ HEPES, $\mathrm{pH}$ 7.5, DNase-RNase-free $\mathrm{H}_{2} \mathrm{O}$ ). For transfection studies, synthetic miR221-3p-23 or miR-221-3p-MUT-23 were annealed with an equimolar amount of the antisense miR-221-3p-AS to form double-stranded miRNA duplexes at $92^{\circ} \mathrm{C}$ for $2 \mathrm{~min}$ and left for $30 \mathrm{~min}$ at room temperature before aliquoting (giving a final concentration of 40 $\mu \mathrm{M})$. In a $10 \mathrm{~cm}$ dish, double-stranded synthetic miR-221 duplexes were reverse-transfected into 300,000 human fibroblasts using $4 \mu \mathrm{L}$ of $4 \mu \mathrm{M}$ miRNA duplexes together with $3.6 \mu \mathrm{L}$ of Lipofectamine RNAiMax in $200 \mu \mathrm{L}$ Opti-MEM, in a final volume of $10 \mathrm{~mL}$ DMEM (with 10\% FBS and $1 \mathrm{mM}$ sodium pyruvate, without antibiotics). The transfected cells were separated into two $10 \mathrm{~cm}$ dishes the following day, and stimulated with IFN- $\beta$ ( $1000 \mathrm{IU} / \mathrm{mL})$ for another $24 \mathrm{~h}$. RNA was extracted and prepared for RT-qPCR and northern blot. The sequences of synthetic miRNAs used are listed in Table 2.

\section{RNA interference (RNAi) experiments}

For PNPT1-RNAi studies, 10,000 or 80,000 MeWo cells per well96-well plate and 12-well plate, respectively-were reversed-transfected with $10 \mathrm{nM}$ of siRNA-RNAiMax mix ( $9 \mu \mathrm{L}$ lipofectamine RNAiMax/ $\mu \mathrm{L}$ siRNA at $40 \mu \mathrm{M}$ ) for $16 \mathrm{~h}$, rinsed with complete RPMI, and stimulated with 1000 IU human IFN- $\beta$ for another 24 h. For western blot analyses of PNPT1, cell lysates were separated at $80 \mathrm{~V}$ on $10 \%$ acrylamide gel for $2 \mathrm{~h}$. Following transfer at $20 \mathrm{~V}$ for $1.5 \mathrm{~h}$ using Bolt Mini Blot Modules (Life Technologies) to Immobilon-FL (Millipore) membranes, membranes were blocked $30 \mathrm{~min}$ in blocking in Odyssey blocking buffer (LI-COR). The membranes were subsequently incubated overnight with 1:1000 rabbit polyclonal anti-PNPT1 (ab157109, Abcam), or rat monoclonal anti- $\beta$-tubulin (ab6160, Abcam). Finally, conjugated secondary antibodies with IRdye800 (Rockland) were used to image the proteins at $800 \mathrm{~nm}$ with an Odyssey scanner (LI-COR).

\section{Nonisotopic northern blot detection of transfected synthetic miRNAs}

Fifteen percent of acrylamide gels were freshly prepared for each run using $5 \times$ TBE Running buffer (ThermoFisher) and UltraPure DNase/ RNase-Free Distilled water (ThermoFisher), and pre-ran in $0.5 \times \mathrm{TBE}$ at $180 \mathrm{~V}$ for $30 \mathrm{~min}$. Six to $10 \mu \mathrm{g}$ of total cellular RNA or $160 \mathrm{pmol}$ of synthetic miR-222-3p-23 and miR-222-3p-20 were mixed in $2 \times$ Novex TBE-Urea sample buffer (ThermoFisher) to a final volume of $20-40 \mu \mathrm{L}$, and denatured at $70^{\circ} \mathrm{C}$ for $5 \mathrm{~min}$. RNAs were separated at $180 \mathrm{~V}$ for $50 \mathrm{~min}$ in $0.5 \times \mathrm{TBE}$. Following transfer at $15 \mathrm{~V}$ for $1.5 \mathrm{~h}$ (at $4^{\circ} \mathrm{C}$ ) using Bolt Mini Blot Modules (ThermoFisher) to GeneScreen Plus nylon membranes (PerkinElmer), membranes were crosslinked at $1200 \mathrm{~kJ}$ in Stratalinker UV Crosslinker (Stratagene), before hybridizing in PerfectHyb Plus Hybridization Buffer (Sigma) for $1 \mathrm{~h}$ at $37^{\circ} \mathrm{C}$ in a rotating oven. Five micrograms of digoxigenin (DIG)-labeled synthetic miR-221-3p probe and U6 probe (synthesized by IDT, see below for the sequences) was added to the hybridization buffer

TABLE 2. Oligonucleotides used in the study

\begin{tabular}{ll}
\hline Name & \multicolumn{1}{c}{ 5'-3' sequence $^{\prime}$} \\
miR-221-3p-20 & rArGrCrUrArCrArUrUrGrUrCrUrGrCrUrGrGrGrU \\
miR-221-3p-23 & rArGrCrUrArCrArUrUrGrUrCrUrGrCrUrGrGrGrUrUrUrC \\
miR-221-3p-MUT-23 & rArGrCrUrArCrArUrUrGrUrCrUrGrCrUrGrGrCrCrGrGrG \\
miR-221-3p-AS & rArUrGrUrArArCrArGrArCrGrArCrC \\
miR-222-3p-21 & rArGrCrUrArCrArUrCrUrGrGrCrUrArCrUrGrGrGrU \\
miR-222-3p-22 & rArGrCrUrArCrArUrCrUrGrGrCrUrArCrUrGrGrGrUrC \\
miR-222-3p-23 & rArGrCrUrArCrArUrCrUrGrGrCrUrArCrUrGrGrGrUrCrU \\
miR-222-3p-24 & rArGrCrUrArCrArUrCrUrGrGrCrUrArCrUrGrGrGrUrCrUrC \\
miR-222-3p-25 & rArGrCrUrArCrArUrCrUrGrGrCrUrArCrUrGrGrGrUrCrUrCrU \\
Probe-U6-DIG & DigN/AAAATATGGAACGCTTCACGATTTGCG/DigN \\
Probe-221-DIG & DigN/ACCAGCAGACAATGTAGCT/DigN \\
h-siPNPT1\#A-S & rGrGrCrArCrUrArArUrArArArGrGrArArUrArArCrUrGrCAT \\
h-siPNPT1\#A-AS & rCrArArArCrGrArUrArArUrCrUrUrCrUrArUrUrUrCrArCrCrCrUrU \\
h-siPNPT1\#B-S & rGrGrGrUrGrArArArUrArGrArArGrArUrUrArUrCrGrUrUTG \\
h-siPNPT1\#B-AS & rCrArArArCrGrArUrArArUrCrUrUrCrUrArUrUrUrCrArCrCrCrUrU \\
siNC5S3 & mCmArUmArUmUrGrCrGrCrGmUrAmUrAmGrUmCrGrCrGrUrUmAG \\
siNC5AS12 & rCrUmArArCrGrCrGmArCmUrAmUrArCrGrCrGrCrArArUmArUmGmGmU \\
\hline
\end{tabular}

Sequences are in 5'-3' (m, 2'OMe modification; r, RNA base and Uppercase, DNA base). DigN/denotes $5^{\prime}$ Digoxigenin (NHS Ester), while /DigN denotes a 3' Digoxigenin (NHS Ester). DIG labeled probes were purified by HPLC. 
and incubated overnight at $37^{\circ} \mathrm{C}$. The following day, the membrane was washed twice for $10 \mathrm{~min}$ in NorthernMax high and low stringency wash buffers (Ambion), before a final wash with wash buffer (0.1 M Maleic acid, $0.15 \mathrm{M} \mathrm{NaCl}, 0.3 \%$ Tween 20, pH 7.5). After 1 $\mathrm{h}$ blocking in Blocking Reagent for nucleic acid hybridization and detection (Roche), the blot was incubated with Anti-Digoxigenin$\mathrm{AP}$ antibody for $1.5 \mathrm{~h}$ at room temperature before being washed twice in wash buffer. For signal detection, BM-Purple Precipitating Substrate (Roche) was applied to the membrane and incubated at room temperature until bands appeared.

\section{Library preparation and RNA sequencing}

Small RNA libraries from human fibroblasts treated or not with IFN- $\beta$ for $24 \mathrm{~h}$ were made using the NEBNext Small RNA Library Prep Set for Illumina (New England Biolabs) according to the manufacturer's instructions and library quality was analyzed using an Agilent Bioanalyzer 2100 (Agilent Technologies). Multiplexed libraries were pooled and spiked with PhiX DNA library (Illumina) at $1 \%$ to ensure a sufficient level of sequence complexity. The libraries were sequenced on a NextSeq 500 machine at the ACRF Cancer Genomics Facility to produce single end, 50 base pair reads.

\section{Bioinformatics analyses of miRNA isoforms}

Raw reads were trimmed to remove adapters and filtered for short sequences using cutadapt v1.3 (Martin 2011) (parameters: minimum read length: $18 \mathrm{nt}$; maximum read length: $50 \mathrm{nt}$; maximum error-rate: 0.2 ; minimum adapter overlap: $5 \mathrm{nt}$ ). The resulting FASTQ files were analyzed and quality checked using FastQC (http://www. bioinformatics.babraham.ac.uk/projects/fastqc/). Reads were mapped against the human reference genome (build hg19) using BWA v0.7.9 (Li and Durbin 2010) using default parameters. IsomiR-level read counts were obtained using a custom python script based on the HTSeq package which uses zero-based genomic coordinates (Anders et al. 2015): alignments from BAM files compared to the genomic locations of microRNAs as specified in miRBase microRNA gene annotation (Kozomara and Griffiths-Jones 2014). For each microRNA, read alignments which overlapped the mature microRNA's genomic locus were classified and counted according to the start and end positions of the alignments. Selective analyses of 3 '-end bases of miR-221-3p and miR-222-3p isoforms were carried out manually using the Integrated Genomics Viewer IGV 2.3_90 (Robinson et al. 2011) and confirmed that templated bases represented $>95 \%$ of $\mathrm{miR}-221-3 \mathrm{p}$ isomiRs between $19-23 \mathrm{nt}$, and $>95 \%$ of miR-222-3p isomiRs 20-24 nt (and that this was not affected by type-I IFN treatment). Read count per million (CPM) normalization was performed by dividing read counts for each isomiR by total counts of all isomiRs $\times 10^{6}$. Small RNA-seq analyses of human fibroblast samples are provided in Supplemental Table S1 ( $3^{\prime}$-isomiRs and $5^{\prime}$-isomiRs with averaged CPM $>1$ in both NT and IFN conditions, separated according to their length). Adapter-trimmed FASTQ files have been deposited in the EBI European Nucleotide Archive (https://www.ebi.ac.uk/ena/data/view/PRJEB22632).

\section{Bioinformatics analyses of $S$. Typhimurium infected DCs}

Reanalysis of genome-wide changes in isomiR expression upon S. Typhimurium (STM) infection shown in Figure 2A and B were carried out as follows: isomiR expression in monocyte-derived DCs from six healthy donors without infection and upon infection for $48 \mathrm{~h}$ was previously profiled by small RNA-seq (Siddle et al. 2015). All preprocessing, alignment, normalization, and filtering steps were performed as described in Siddle et al. (2015). Only canonical and templated $3^{\prime}$ isomiRs were retained for further analysis. In addition, all templated $3^{\prime}$ isomiRs aligning to miRNAs for which templated $3^{\prime}$ isomiRs accounted for $<75 \%$ of all reads aligning to the miRNA were removed. After these filtering steps, we obtained 1001 isomiRs between 18 and $26 \mathrm{nt}$ in length, from 132 mature miRNAs (Supplemental Table S2). IsomiRs were classified by length and mean expression counts across all six individuals were compared between noninfected and STM-infected cells at each time-point. Data from Figure 2C were generated by reanalyzing Supplemental Table S8 from Siddle et al. (2015), listing isomiRs expressed in monocytes-derived DCs (filtering out isoforms accounting for $<5 \%$ of the total reads), with fold changes of their differential expression upon infection compared to noninfected samples. Only templated $3^{\prime}$ isomiRs were retained for further analysis, for a total of 774 miRNA isoforms. Relative $\log _{2}$ fold changes for each miRNA family were calculated by subtracting the row mean, and dividing by row standard deviation. Clustering of miRNA rows was carried out using one minus Pearson correlation.

\section{Statistical analyses}

Statistical analyses were carried out using Prism 7 (GraphPad Software Inc.). Two-tailed unpaired $t$-tests and nonparametric Mann-Whitney $U$-tests were used to compare pairs of conditions, when appropriate. Symbols used: ${ }^{*} P \leq 0.05,{ }^{* *} P \leq 0.01,{ }^{* * *} P \leq$ $0.001,{ }^{* * * *} P \leq 0.0001$.

\section{SUPPLEMENTAL MATERIAL}

Supplemental material is available for this article.

\section{ACKNOWLEDGMENTS}

We thank P.J. Hertzog and N.A. de Weerd for providing murine recombinant IFN- $\beta$, M.A. Behlke for RNA synthesis, X. Zhang and $\mathrm{V}$. Hornung for human melanoma and fibroblast cell lines, respectively, and R.E. Smith for help with the redaction of this paper. We acknowledge the Monash Health Translational Precinct Research Platforms for access to the RT-qPCR instruments. This work was funded in part by the Australian National Health and Medical Research Council (1062683 to M.P.G., 1069128 to C.P.B. and G.J. G., and fellowship 1026191 to G.J.G.); the Australian Research Council (140100594 Future Fellowship to M.P.G.); the Canadian Fonds de recherche du Québec - Santé (35071 FRSQ Fellowship to G.P.); and the Victorian Government's Operational Infrastructure Support Program. The laboratory of L.Q.M. is funded by the European Research Council under the European Union's Seventh Framework Programme (FP/2007-2013)/ERC grant agreement no. 281297.

Received October 19, 2017; accepted December 18, 2017. 


\section{REFERENCES}

Anders S, Pyl PT, Huber W. 2015. HTSeq-a Python framework to work with high-throughput sequencing data. Bioinformatics 31: 166-169.

Das SK, Sokhi UK, Bhutia SK, Azab B, Su ZZ, Sarkar D, Fisher PB. 2010. Human polynucleotide phosphorylase selectively and preferentially degrades microRNA-221 in human melanoma cells. Proc Natl Acad Sci 107: 11948-11953.

Ferrand J, Gantier MP. 2016. Assessing the inhibitory activity of oligonucleotides on TLR7 sensing. Methods Mol Biol 1390: 79-90.

Galardi S, Mercatelli N, Farace MG, Ciafre SA. 2011. NF-kB and c-Jun induce the expression of the oncogenic miR-221 and miR-222 in prostate carcinoma and glioblastoma cells. Nucleic Acids Res 39: 3892-3902.

Gantier MP, McCoy CE, Rusinova I, Saulep D, Wang D, Xu D, Irving AT, Behlke MA, Hertzog PJ, Mackay F, et al. 2011. Analysis of microRNA turnover in mammalian cells following Dicer1 ablation. Nucleic Acids Res 39: 5692-5703.

Ha M, Kim VN. 2014. Regulation of microRNA biogenesis. Nat Rev Mol Cell Biol 15: 509-524.

Juzenas S, Venkatesh G, Hubenthal M, Hoeppner MP, Du ZG, Paulsen M, Rosenstiel P, Senger P, Hofmann-Apitius M, Keller A, et al. 2017. A comprehensive, cell specific microRNA catalogue of human peripheral blood. Nucleic Acids Res 45: 9290-9301.

Karali M, Persico M, Mutarelli M, Carissimo A, Pizzo M, Singh Marwah V, Ambrosio C, Pinelli M, Carrella D, Ferrari S, et al. 2016. High-resolution analysis of the human retina miRNome reveals isomiR variations and novel microRNAs. Nucleic Acids Res 44: 1525-1540.

Kaur S, Sassano A, Dolniak B, Joshi S, Majchrzak-Kita B, Baker DP, Hay N, Fish EN, Platanias LC. 2008. Role of the Akt pathway in mRNA translation of interferon-stimulated genes. Proc Natl Acad Sci 105: 4808-4813.

Kozomara A, Griffiths-Jones S. 2014. miRBase: annotating high confidence microRNAs using deep sequencing data. Nucleic Acids Res 42: D68-D73.

Landgraf P, Rusu M, Sheridan R, Sewer A, Iovino N, Aravin A, Pfeffer S, Rice A, Kamphorst AO, Landthaler M, et al. 2007. A mammalian microRNA expression atlas based on small RNA library sequencing. Cell 129: 1401-1414.

Li H, Durbin R. 2010. Fast and accurate long-read alignment with Burrows-Wheeler transform. Bioinformatics 26: 589-595.

Liu S, Sun X, Wang M, Hou Y, Zhan Y, Jiang Y, Liu Z, Cao X, Chen P, Liu Z, et al. 2014. A microRNA 221- and 222-mediated feedback loop maintains constitutive activation of NFKB and STAT3 in colorectal cancer cells. Gastroenterology 147: 847-859.e11.

Magee R, Telonis AG, Cherlin T, Rigoutsos I, Londin E. 2017. Assessment of isomiR discrimination using commercial $\mathrm{qPCR}$ methods. Noncoding RNA 3: p18.

Martin M. 2011. Cutadapt removes adapter sequences from highthroughput sequencing reads. EMBnetJournal 17: 10-12.
Neilsen CT, Goodall GJ, Bracken CP. 2012. IsomiRs-the overlooked repertoire in the dynamic microRNAome. Trends Genet 28: 544-549.

O'Neill LA, Sheedy FJ, McCoy CE. 2011. MicroRNAs: the fine-tuners of Toll-like receptor signalling. Nat Rev Immunol 11: 163-175.

Robinson JT, Thorvaldsdottir H, Winckler W, Guttman M, Lander ES, Getz G, Mesirov JP. 2011. Integrative genomics viewer. Nat Biotechnol 29: 24-26.

Robinson N, McComb S, Mulligan R, Dudani R, Krishnan L, Sad S. 2012. Type I interferon induces necroptosis in macrophages during infection with Salmonella enterica serovar Typhimurium. Nat Immunol 13: 954-962.

Rusinova I, Forster S, Yu S, Kannan A, Masse M, Cumming H, Chapman R, Hertzog PJ. 2013. Interferome v2.0: an updated database of annotated interferon-regulated genes. Nucleic Acids Res 41: D1040-D1046.

Schamberger A, Orban TI. 2014. 3' IsomiR species and DNA contamination influence reliable quantification of microRNAs by stem-loop quantitative PCR. PLoS One 9: e106315.

Sehgal M, Zeremski M, Talal AH, Ginwala R, Elrod E, Grakoui A, Li QG, Philip R, Khan ZK, Jain P. 2015. IFN- $\alpha$-induced downregulation of miR-221 in dendritic cells: implications for HCV pathogenesis and treatment. J Interferon Cytokine Res 35: 698-709.

Siddle KJ, Tailleux L, Deschamps M, Loh YH, Deluen C, Gicquel B, Antoniewski C, Barreiro LB, Farinelli L, Quintana-Murci L. 2015. Bacterial infection drives the expression dynamics of microRNAs and their isomiRs. PLoS Genet 11: e1005064.

Stifter SA, Gould JA, Mangan NE, Reid HH, Rossjohn J, Hertzog PJ, de Weerd NA. 2014. Purification and biological characterization of soluble, recombinant mouse IFN $\beta$ expressed in insect cells. Protein Expr Purif 94: 7-14.

Tan GC, Chan E, Molnar A, Sarkar R, Alexieva D, Isa IM, Robinson S, Zhang S, Ellis P, Langford CF, et al. 2014. 5' isomiR variation is of functional and evolutionary importance. Nucleic Acids Res 42: 9424-9435.

Telonis AG, Magee R, Loher P, Chervoneva I, Londin E, Rigoutsos I. 2017. Knowledge about the presence or absence of miRNA isoforms (isomiRs) can successfully discriminate amongst 32 TCGA cancer types. Nucleic Acids Res 45: 2973-2985.

Wu H, Neilson JR, Kumar P, Manocha M, Shankar P, Sharp PA, Manjunath N. 2007. miRNA profiling of naïve, effector and memory CD8 T cells. PLoS One 2: e1020.

Yamamoto M, Sato S, Hemmi H, Hoshino K, Kaisho T, Sanjo H, Takeuchi O, Sugiyama M, Okabe M, Takeda K, et al. 2003. Role of adaptor TRIF in the MyD88-independent toll-like receptor signaling pathway. Science 301: 640-643.

Yu F, Pillman KA, Neilsen CT, Toubia J, Lawrence DM, Tsykin A, Gantier MP, Callen DF, Goodall GJ, Bracken CP. 2017. Naturally existing isoforms of miR-222 have distinct functions. Nucleic Acids Res 45: 11371-11385. 

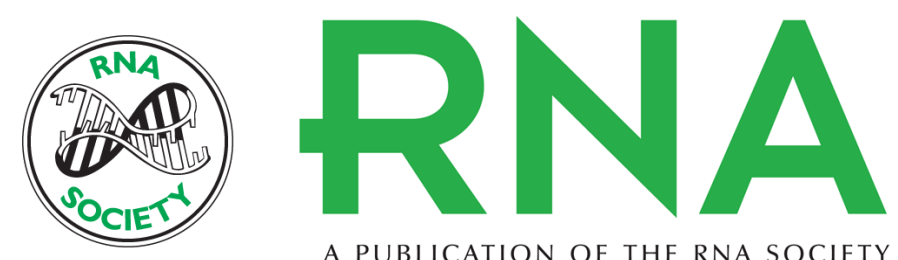

A PUBLICATION OF THE RNA SOCIETY

\section{miR-222 isoforms are differentially regulated by type-I interferon}

Charlotte Nejad, Katherine A. Pillman, Katherine J. Siddle, et al.

RNA 2018 24: 332-341 originally published online December 20, 2017

Access the most recent version at doi:10.1261/rna.064550.117

Supplemental Material

References

Creative Commons License

Email Alerting Service
http://rnajournal.cshlp.org/content/suppl/2017/12/20/rna.064550.117.DC1

This article cites 28 articles, 3 of which can be accessed free at: http://rnajournal.cshlp.org/content/24/3/332.full.html\#ref-list-1

This article is distributed exclusively by the RNA Society for the first 12 months after the full-issue publication date (see http://rnajournal.cshlp.org/site/misc/terms.xhtml). After 12 months, it is available under a Creative Commons License (Attribution-NonCommercial 4.0 International), as described at http://creativecommons.org/licenses/by-nc/4.0/.

Receive free email alerts when new articles cite this article - sign up in the box at the top right corner of the article or click here.

To subscribe to RNA go to:

http://rnajournal.cshlp.org/subscriptions 